\title{
BMJ Open The impact of distance and duration of travel on participation rates and participants' satisfaction: results from a pilot study at one study centre in Pretest 2 of the German National Cohort
}

Aparna Schweitzer, ${ }^{1}$ Manas K Akmatov, ${ }^{1,2}$ Florentina Kindler, ${ }^{1}$ Yvonne Kemmling, ${ }^{1}$ Lothar Kreienbrock, ${ }^{3}$ Gérard Krause, ${ }^{1,4}$ Frank Pessler ${ }^{1,2}$

To cite: Schweitzer A, Akmatov MK, Kindler F, et al. The impact of distance and duration of travel on participation rates and participants' satisfaction: results from a pilot study at one study centre in Pretest 2 of the German National Cohort. BMJ Open 2015;5: e007461. doi:10.1136/ bmjopen-2014-007461

- Prepublication history for this paper is available online. To view these files please visit the journal online (http://dx.doi.org/10.1136/ bmjopen-2014-007461)

Received 15 December 2014 Revised 30 May 2015 Accepted 15 June 2015

CrossMark

For numbered affiliations see end of article.

Correspondence to Dr Aparna Schweitzer; aparna.schweitzer@dzne.de

\section{ABSTRACT}

Objectives: In this pilot study within the Pretest 2 phase of the German National Cohort, we aimed to (1) test the hypothesis that distance and duration of travel to a study centre may affect participation rates and participants' satisfaction and (2) to obtain data that would help to select recruitment areas around the study centre Hannover with the greatest projected participation rate for the main study.

Setting: Mixed urban/suburban environment in Northern Germany with approximately 600000 inhabitants. 4 recruitment areas with divergent estimated mean distances (range, 7-40 km) and duration of travel to the study centre Hannover were selected.

Participants: 1050 men and women (ratio, 1:1), aged 20-69 years, were randomly selected from the population registries of the 4 recruitment areas and invited by mail to participate in the Pretest 2 study programme at the study centre Hannover, covering a variety of questionnaire-based and physical assessments. 166 individuals participated (16\%). Interventions: All 166 participants completed a travel questionnaire containing 5 items relating to travel duration and satisfaction, amounting to a participation rate of $100 \%$ in the questionnaire-based part of the study.

Results: Participation rates in the Pretest 2 programme at the study centre Hannover by area ranged from $11 \%$ (area farthest from the study centre, estimated median distance $38 \mathrm{~km}$ ) to $18 \%$ (nearest area, $2 \mathrm{~km}$ ). The odds of non-participation were highest in the area farthest from the study centre (adjusted OR 2.06; $\mathrm{p}=0.01 ; \mathrm{Cl}$ 1.28 to 3.32). Nonetheless, $97 \%$ of participants were satisfied with travel duration.

Conclusions: Increasing distance was associated with a lower participation rate. However, acceptance of duration of travel was high, irrespective of distance or duration. Thus, recruiting in farther away locations may select individuals with a greater frustration tolerance for travel to the study centre, perhaps due to a greater interest in participating in health-oriented studies and thus different health-related behaviour.

\section{Strengths and limitations of this study}

- To the best of our knowledge, this is one of the first studies to investigate the role of distance and duration of travel on participation rates and satisfaction in a population-based study.

- Despite the small sample size, we identified a significantly lower response at the locations farthest away from the study centre.

- One of the limitations of this study is the small sample size, accompanied by the low participation rate for the overall Pretest 2 at this study centre $(16 \%$, 166/1050). However, the low response rate was comparable to those at the other 17 study centres participating in Pretest 2 across the nation.

- We are now proceeding with the main study. The recruitment area for the study was limited to Hannover city due to a variety of reasons including the results of this study.

\section{BACKGROUND}

Longitudinal cohort studies are important for studying aetiological mechanisms underlying population-based and individual differences in the incidence of disease. ${ }^{12}$ The German National Cohort (GNC) is a large-scale, nationwide, prospective population-based study that aims at recruiting a representative sample from the general population in order to investigate aetiology, risk factors and effective preventive strategies for common chronic and infectious diseases. ${ }^{34}$

Participation in epidemiological studies has been declining over the past 30 years, with even steeper declines seen in recent years. ${ }^{5-9}$ In order to counteract this trend, it is important to identify any factors that may reduce participation rates. Distance and duration of travel to a study centre and the modes of 
transportation used appear to be obvious entities in this regard. ${ }^{10}{ }^{11}$ However, surprisingly few studies have addressed their impact, if any, on participation or satisfaction associated with population-based studies. A Japanese population-based genetic cohort study ${ }^{11}$ reported a significant association between the convenience of survey location and participation rates: in participants with a "convenient location" (survey locations that were as near as possible to the residential areas of participants), there were significantly higher participation rates (OR, 1.3; 95\% CI 1.20 to 1.35 ) than for those from farther away locations. Recruitment strategies and examination procedures of the GNC were tested in 2012 in a variety of pilot studies conducted during the Pretest 2 phase, featuring 18 recruitment centres across the nation. The present pilot study was conducted at one centre, the Study Centre Hannover of the Helmholtz Centre for Infection Research. It primarily aimed to assess the impact of distance and duration of travel on participation rates and participants' satisfaction in order to delineate the local recruitment area for the main study of the GNC (to start in 2014) with the greatest projected response rate. A secondary aim was to assess the potential, if any, of several incentives to the participants to increase willingness to participate in future studies at this location.

\section{METHODS}

\section{Selection of recruitment areas}

In the Pretest 2 phase of the GNC, each of the 18 study centres across the nation, including the Study Centre Hannover, was to recruit approximately 200 individuals in order to test preliminary recruitment strategies and study protocols. Since this number was fixed across all study centres, a power calculation for the presented pilot study was not done. We targeted four recruitment areas, Hannover city, Isernhagen, Lehrte and Neustadt am Rübenberge (referred to as Neustadt henceforth), to address the aforementioned questions. The four recruitment areas were selected in such a way that they differed both in distance from the study centre and in the anticipated time required for travel by public or personal transportation. Estimated mean distances and convenience of access by car or public transportation were as follows: Hannover city, $7 \mathrm{~km}$ with good roadway connections and broadly available public transport connections to the study centre (no or only one transfer required from most locations); Isernhagen, $15 \mathrm{~km}$ with good roadway connections but less convenient availability of public transport (at least two transfers required); Lehrte, $21 \mathrm{~km}$ with good roadway and public transportation connections (one transfer required) and Neustadt, $40 \mathrm{~km}$ with good yet long road connections and the least convenient public transport connectivity (longest distance, at least two transfers required).

\section{Sociodemographic characteristics of the recruitment areas}

On the basis of national census data, average age was slightly lower in Hannover (42.3 years), Lehrte (43.3 years) and Neustadt (43.3 years) than in Isernhagen (45 years). There were slightly more women $(\geq 50 \%)$ than men $(\leq 49 \%)$ in all four areas, which also reflects national estimates. The proportion of migrants was highest in Hannover (30.6\%), followed by Isernhagen (18.2\%), Lehrte $(17.3 \%)$ and Neustadt $(13.9 \%)$. Income has been associated with the likelihood of participating in a health study. ${ }^{12}$ Data on income were not available, but the unemployment rate was lowest in Isernhagen $(1.6 \%)$ followed by Neustadt $(1.7 \%)$, Lehrte $(2 \%)$ and Hannover $(3.5 \%)$ (national average, $2.5 \%) \cdot{ }^{13}$ In terms of education, the proportion of university graduates was highest in Hannover (13.7\%), followed by Isernhagen (11.5\%), Lehrte $(5.8 \%)$ and Neustadt $(5.6 \%)$. Of these variables, the differences in migration status were most likely to affect the likelihood of participating in health-oriented studies.

\section{Recruitment and travel questionnaire}

All individuals who were between the ages of 20-69 years and had their principal residence in one of the aforementioned areas were eligible to participate in Pretest 2 at the Study Centre Hannover. Individuals to be invited were selected by random sampling using local population registries. In the study centre, the participants were scheduled to complete a panel of interviews and questionnaires, undergo medical examinations and provide a variety of biosamples. A separate informed consent was obtained for participating in the travel questionnaire study, and participants were informed that the data collected would be used for future planning of recruitment for the GNC. Participants who provided informed consent to complete the travel questionnaire were given the questionnaire for self-completion in the study centre. The questionnaire covered five principal areas: mode of transportation used, duration of travel, acceptability of duration of travel, satisfaction with travel conditions, and hypothetical travel incentives that would motivate participants to take part in a hypothetical future study conducted at this study centre. It contained several closed-ended items as well as open-ended questions. The items were measured on a Likert scale when indicated.

\section{Statistical analyses}

Distances from the participants' residences to the study centre were computed with Google maps (https://maps. google.com/) using area postal codes, as using addresses was not possible for reasons of privacy and data protection. Participation rates were calculated by dividing the number of individuals who participated by the total number of individuals contacted. In order to test whether the differences in travel duration were significant, a travel duration score was generated by transforming the self-reported time frames into integers as follows: $1=<15 \mathrm{~min}, \quad 2=15-29 \mathrm{~min}, 3=30-44 \mathrm{~min}, \quad 4=45-59 \mathrm{~min}$, $5=60-89 \mathrm{~min}$ and $6=\geq 90 \mathrm{~min}$. As these data were measured on an ordinal scale and were not normally distributed, the Mann-Whitney $U$ test was used to test for 
differences in median travel duration score between Hannover city and each of the three other areas. Since data on age, sex and citizenship were available for both participants and non-participants, age-adjusted, sexadjusted and citizenship-adjusted odds ratios (ORs) and 95\% CIs for non-participation with respect to location were calculated as measures of the strength of association. We also assessed sociodemographic differences among the four areas applying Fisher's exact test (to examine the significance of the association (contingency) between categorical variables) or the Kruskal-Wallis test (to assess differences in age across groups). Analyses were done using Stata V.12.1 for Windows (StataCorp LP, Texas, USA) and SPSS for Windows, V.19 (IBM Corporation, Armonk, New York, USA).

\section{RESULTS}

Between September and December 2012, 1050 individuals were contacted by mail. The overall participation rate in Pretest 2 at the Study Centre Hannover was 16\% $(166 / 1050)$. All participants at this study centre took part in the travel questionnaire survey. The median age of the participants was 52 years (IQR 43-61). The male-to-female ratio was $1: 1$. The participation rate was highest among those aged 60-69 years (19\%; 50/269) and lowest among those aged 20-29 years (11\%, 12/ 105). Across recruitment areas, the participation rate was highest in Hannover city $(18 \%)$, slightly lower in Lehrte $(17 \%)$ and Isernhagen $(16 \%)$, and by far the lowest in Neustadt $(11 \%)$, the region farthest away from the study centre (figure 1). Table 1 depicts sociodemographic characteristics of the participants. We did not find any statistically significant differences among the four areas in terms of the available sociodemographic variables (table 1$)$.

\section{Distance and self-reported duration of travel to the study centre}

Figure 1 shows, for each of the four recruitment areas, the median distance from the participants' residences to the study centre and the self-reported duration of travel to the study centre. The median distance between the residences of all participants and the study centre was $16 \mathrm{~km}$ (IQR 2-21), but, as expected, it varied significantly among the four regions, ranging from $2 \mathrm{~km}$ (Hannover city) to $38 \mathrm{~km}$ (Neustadt). The most

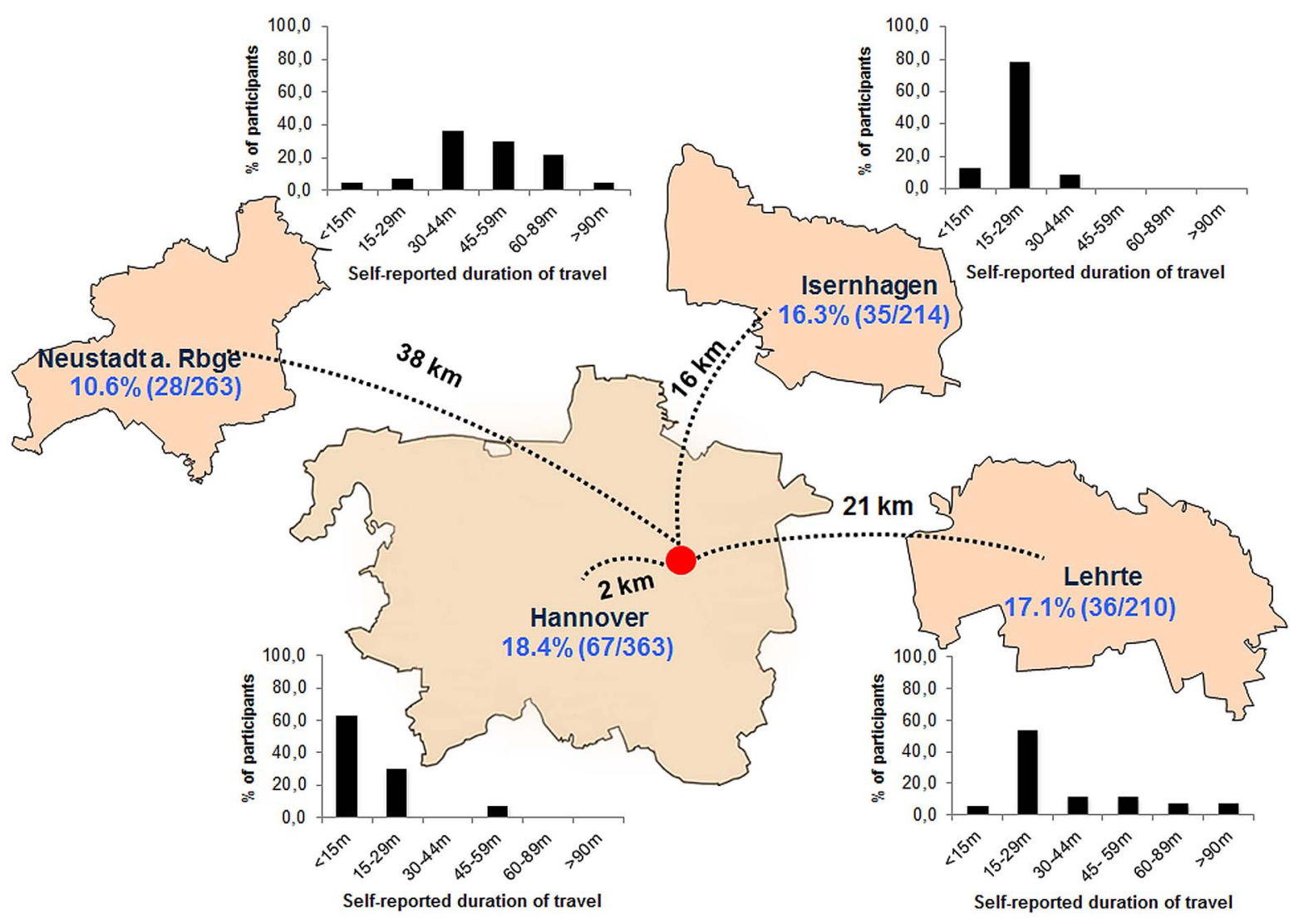

Figure 1 Map of the recruitment areas, showing median distances, self-reported duration of travel to the study centre and participation rates. Median distances from the participants' residences were estimated with Google Maps using postal codes of the participants' addresses. Participation rates (blue font) were calculated by dividing the total number of participants from each area by the total number of people invited from each area. Duration of travel was self-reported by the participants. The bar charts show frequencies with which the participants from each of the four recruitment areas reported the six time categories of travel duration plotted along the $\mathrm{x}$-axis. 
Table 1 Sociodemographic characteristics of the participants*

\begin{tabular}{|c|c|c|c|c|c|c|}
\hline Variables* & All n (\%) & Hannover city n (\%) & Isernhagen $\mathbf{n}(\%)$ & Lehrte n (\%) & Neustadt n (\%) & p Valuet \\
\hline Invited persons & 1050 & 363 & 214 & 210 & 263 & \\
\hline Participation rate & $166(15.8)$ & $67(18.4)$ & 35 (16.3) & $36(17.1)$ & $28(10.6)$ & \\
\hline Median age (IQR) & $51.5(43.3-61)$ & $51(43-62)$ & $52(43-61)$ & $49.5(42.5-57)$ & $53(46.3-61.8)$ & $0.70 \ddagger$ \\
\hline \multicolumn{7}{|l|}{ Sex } \\
\hline Male & $83(50)$ & $38(56.7)$ & $12(34.2)$ & $20(55.5)$ & $13(46.5)$ & 0.18 \\
\hline Female & $83(50)$ & $29(43.2)$ & $23(65.7)$ & $16(44.4)$ & $15(53.5)$ & \\
\hline \multicolumn{7}{|l|}{ Country of birth } \\
\hline Germany & $144(86.7)$ & $55(82)$ & $31(88.5)$ & 32 (89) & $26(92.8)$ & 0.26 \\
\hline Other & $20(12)$ & 12 (17.9) & $4(11.4)$ & $2(5.5)$ & $2(7.1)$ & \\
\hline Missing values & 2 (1.2) & - & - & $2(5.5)$ & - & \\
\hline \multicolumn{7}{|l|}{ Education level } \\
\hline Low & $60(36.1)$ & $29(43.2)$ & $13(37.1)$ & $10(27.7)$ & $8(28.6)$ & 0.50 \\
\hline Middle & $73(43.9)$ & 25 (37.3) & $15(42.8)$ & $18(50)$ & $15(53.6)$ & \\
\hline Higher & $4(2.4)$ & $1(1.5)$ & $0(0)$ & $1(2.8)$ & $2(7.1)$ & \\
\hline Missing values & $29(17.5)$ & $12(17.9)$ & $7(20)$ & 7 (19.4) & 3 (10.7) & \\
\hline
\end{tabular}

frequently self-reported duration of travel also varied across the four regions in that it was $<15 \mathrm{~min}$ in Hannover city, $<30 \mathrm{~min}$ in Lehrte and Isernhagen, and $<45$ min in Neustadt (figure 1). As expected, longer distances of travel were associated with longer self-reported travel durations, both values being lowest in Hannover city, intermediate in Lehrte and Isernhagen, and highest in Neustadt. The differences in median travel duration score between Hannover city and each of the three other areas were significant: Hannover city versus Isernhagen, $\mathrm{p}=0.0002$; Hannover city versus Lehrte, $\mathrm{p}<0.0001$; and Hannover city versus Neustadt, $\mathrm{p}<0.0001$. The results of the multivariable logistic regression analysis (adjusted for sex, age and citizenship) showed that the odds of non-participation compared with Hannover city were significantly higher only in Neustadt, that is, the area with the largest distance, longest self-reported travel duration and highest travel duration score of participants, but not in Lehrte or Isernhagen (table 2). Since data matching the national census definition of migration status were not recorded, citizenship was used as a proxy indicator of migration status. Of note, individuals with non-German citizenship were significantly more likely to be non-participants than those with German citizenship, but owing to the relatively low proportion of non-German nationals in all four areas (Hannover city $10 \%$, Isernhagen $6.6 \%$, Lehrte $5.7 \%$, Neustadt $5.3 \%$ ), the overall effect of citizenship on participation rates was minor (table 2, compare columns 3 and 4).

\section{Mode of transportation}

The majority of participants travelled by car (table 3). There was no significant association between age or sex and mode of transportation used. There were some differences in transportation used across areas of residence in that all participants from Isernhagen used cars compared with only $61 \%$ of the participants from Hannover city. A majority of participants $(97 \%(159 / 164))$ found the duration of travel acceptable irrespective of the area of residence or start location. However, acceptability of duration of travel did decrease somewhat with the duration of travel $(\mathrm{p}<0.0001)$.

Table 2 Adjusted ORs for the likelihood of non-participation

Unadjusted OR (95\% Cl)

Adjusted OR* $(95 \% \mathrm{Cl})$

Adjusted OR† $(95 \% \mathrm{Cl})$

\section{Region}

Isernhagen vs Hannover

Lehrte vs Hannover

Neustadt vs Hannover

Age (change per year)

Sex

Male vs female

Citizenship

German vs non-German
1.14 (0.73 to 1.78$)$

$1.12(0.71$ to 1.75$)$

1.94 (1.21 to 3.11$)$

0.99 (0.98 to 1.01 )

1.02 (0.73 to 1.42$)$

0.27 (0.10 to 0.74$)$
1.17 (0.74 to 1.84$)$

$1.10(0.70$ to 1.73$)$

$1.96(1.22$ to 3.15$)$

0.99 (0.98 to 1.01 )

1.02 (0.73 to 1.42$)$
1.21 (0.77 to 1.90$)$

1.15 (0.73 to 1.81$)$

2.06 (1.28 to 3.32$)$

0.99 (0.98 to 1.01 )

1.01 (0.72 to 1.41$)$

${ }^{*}$ Adjusted for sex and age.

†Adjusted for sex, age and citizenship. 
Table 3 Mode of transportation used by participants in Pretest 2 of the German National Cohort to reach the study centre Hannover

\begin{tabular}{|c|c|c|c|c|c|}
\hline \multirow[b]{2}{*}{ Variables } & \multirow[b]{2}{*}{$\mathbf{N}$} & \multicolumn{3}{|c|}{ Transportation used } & \multirow[b]{2}{*}{ p Valuef } \\
\hline & & $\overline{\text { Car (\%) }}$ & Public transport (\%) & Other* $(\%)$ & \\
\hline Overall & $155 \ddagger$ & 76 & 9.0 & 16 & \\
\hline Sex & & & & & 0.28 \\
\hline Male & 77 & 74 & 6.5 & 20 & \\
\hline Female & 78 & 77 & 12 & 12 & \\
\hline Age group (years) & & & & & 0.26 \\
\hline $20-29$ & 11 & 64 & 27 & 9.1 & \\
\hline 30-39 & 14 & 86 & 7.1 & 7.1 & \\
\hline $40-49$ & 43 & 74 & 2.3 & 23 & \\
\hline $50-59$ & 40 & 73 & 10 & 18 & \\
\hline $60-69$ & 47 & 77 & 11 & 11 & \\
\hline Residence & & & & & $<0.0001$ \\
\hline Hannover & 62 & 61 & 3.2 & 36 & \\
\hline Isernhagen & 34 & 100 & 0 & 0 & \\
\hline Lehrte & 31 & 81 & 16 & 3.2 & \\
\hline Neustadt & 28 & 71 & 25 & 3.6 & \\
\hline
\end{tabular}

\section{Hypothetical incentives}

Only 59 participants answered the question about hypothetical travel incentives "I would only participate in a future study if provided with ...". 39 of them (66\%) did not think that incentives would encourage their participation in a future study conducted at this study centre. Those who did answer affirmatively considered the following incentives to be effective: reimbursement of travel costs (in addition to the remuneration for participating in the study), $34 \%(19 / 56)$; availability of a reserved parking space, $29 \%(15 / 51)$; provision of a bus or train network plan, $20 \%(12 / 59)$; a shuttle bus from the train or bus station to the study centre, $12 \%(7 / 59)$; and provision of a taxi voucher, $10 \%(6 / 59)$.

\section{DISCUSSION \\ Effects of distance and duration of travel on participation rates}

In this pilot study within the GNC, a negative association between distance and duration of travel to the study centre and participation rates became evident. Our findings concur with the few available relevant published studies. ${ }^{10}{ }^{11}$ Interestingly, there appeared to be a threshold effect, that is, a certain travel distance and/or duration to the study centre below which participation rates in the recruitment areas were fairly comparable, as participation rates did not differ significantly among Hannover city, Isernhagen and Lehrte. It is not easy to explain this observation, but it may relate to the fact that, as opposed to Isernhagen and Lehrte, Neustadt is located on the opposite side of the city centre of Hannover (west) as the study centre (east), meaning that prospective participants might have been more reluctant to travel to the study centre because they would have had to either pass through the city centre or take peripheral motorways. The overall results of this study must be interpreted with caution as they are based on the assumption that non-participants would have taken the same time to travel as participants. Time taken to travel depends on the choice of transportation and also location within a region, that is, Hannover city versus a suburban area in Hannover, and we do not have information about either for non-participants in this study. For instance, it is possible that non-participants decided against participating in the study because of lack of a car or because they resided in locations situated farther away from where participants had to travel. Indeed, a car was a significantly more frequently used mode of transportation in the three areas other than Hannover city, and self-reported duration of travel was longer among those participants who used public transportation. Several postal codes were available for Hannover city, but there was only one code each for Lehrte, Isernhagen and Neustadt. Therefore, it was not possible to differentiate travel distances among the participants from any one of the latter three areas. On the basis of our data, the observed differences in participation rates among the four areas could not be explained by age, sex, country of birth, citizenship or education level (tables 1 and 2). Consistent with previous reports that the willingness to participate in population-based surveys in Germany is lower among individuals with an immigrant background as compared to non-migrants, ${ }^{14-16}$ citizenship (which was used as a proxy for migration status) did affect the likelihood of participation strongly. However, it did not have a notable effect on overall participation rates due to the relatively low proportion of non-German citizens across the four areas. The criterion "citizenship" alone might not be the best 
indicator of migration status. Additional variables such as country of birth of the individual and/or either parent, for non-participants, would have enabled a more thorough assessment of migration status. However, this information was not available for non-participants.

\section{Participants' satisfaction with travel conditions (parking spaces, sign posting to the study centre)}

Of note, satisfaction with travel conditions was high, which might be accounted for by a high intrinsic personal motivation to participate in the GNC. Indeed, an altruistic motivation for participating in cohort studies has been reported in previous studies. ${ }^{17}$ Although participants gave high ratings with respect to travel conditions, based on the qualitative analysis of comments and suggestions for improvement, the need for more parking spaces at the study centre became clear. In addition, some participants expressed that "better signposting would be necessary to make the centre easy to locate". The travel directions document provided to the participants prior to their scheduled appointment to the study centre also needed improvement, particularly with respect to the map showing directions to the study centre.

\section{Role of hypothetical incentives}

Participants of the Pretest 2 phase of the GNC had to travel to the study centre solely for the study. Winkler et $a l^{16}$ reported results of recruitment and response rates in feasibility studies conducted in 2011 during Pretest 1 of the GNC and found that offering results from medical examinations had a positive effect on recruitment. In addition, offering monetary incentives also seemed to improve response. At the Study Center Hannover, participants in the Pretest 2 phase were offered a $€ 20$ cash incentive for participation. The fact that only $36 \%$ of the participants responded to the question on travel incentives would suggest that a majority were satisfied with this remuneration and did not expect any additional incentives, most likely because of an altruistic motivation or an intrinsically high personal motivation to be a part of this study. However, our study cannot be used to fully assess the role of incentives, as questions regarding incentives should have been posed to non-participants, too. Unfortunately, this was not possible in the context of the standardised non-responder questionnaire used in Pretest 2 of the GNC.

\section{CONCLUSIONS}

Increasing distance and self-reported duration of travel to the study centre were associated with lower participation rates once a certain threshold was exceeded. However, these factors did not markedly affect participants' travel satisfaction. An intrinsically high motivation among participants might account for this. More intensive recruitment and alternative recruitment strategies, such as offering additional incentives, may be necessary in remote areas to achieve participation rates comparable to areas located closer to the study centre. However, overly aggressive recruitment may lead to the inclusion of reluctant participants at baseline who are more likely to drop out during follow-up. Lower retention rates would compromise the validity of a prospective study. Using mobile study centres to recruit in remote areas could perhaps circumvent such obstacles, but this was ruled out in the GNC because of budgetary constraints. Considering these issues, Neustadt was considered unsuitable early on for recruitment for the GNC. In addition, Lehrte and Isernhagen were excluded due to other aspects of recruitment logistics and delineation of the recruitment area.

\section{Author affiliations}

${ }^{1}$ Department of Epidemiology, Helmholtz Centre for Infection Research, Braunschweig, Lower Saxony, Germany

${ }^{2}$ TWINCORE Centre for Experimental and Clinical Infection Research, Hannover, Germany

${ }^{3}$ Institute for Biometry, Epidemiology and Information Processing, University of Veterinary Medicine, Hannover, Germany

${ }^{4}$ Hannover Medical School, Hannover, Germany

Acknowledgements The authors would like to extend their gratitude to staff members of the Study Center Hannover, in particular Ms Fabienne Schulz, Ms Sabrina Wieghold and Mr Martin Andrzejak for their contributions in planning and conducting the study.

Contributors AS conducted the statistical analysis and drafted the manuscript. LK and FP conceived and designed the study. MKA participated in the statistical analysis. MKA and FP provided a critical review of the manuscript drafts. GK provided intellectual input into subsequent manuscript drafts and critically reviewed the manuscript. FK and YK made substantial contributions to the acquisition of data and their critical revision. All authors approved of the version to be published. AS, MKA and FP had full access to all of the data in the study and take responsibility for the integrity of the data and the accuracy of the data analysis.

Funding This project was conducted in the context of the pretest studies of the German National Cohort (http://www.nationale-kohorte.de). These were funded by the German Federal Ministry of Education and Research (BMBF), grant no. (Förderkennzeichen) 01ER1001A-I, and supported by the Helmholtz Association as well as by the participating universities and Institutes of the Leibniz Association. Data analysis and preparation of the manuscript were also supported by the Helmholtz Association's Cross-Programme Initiative in individualised Medicine, iMed.

\section{Competing interests None declared.}

Patient consent Obtained.

Ethics approval State Board of Physicians of the Federal State of Lower Saxony.

Provenance and peer review Not commissioned; externally peer reviewed.

Open Access This is an Open Access article distributed in accordance with the Creative Commons Attribution Non Commercial (CC BY-NC 4.0) license, which permits others to distribute, remix, adapt, build upon this work noncommercially, and license their derivative works on different terms, provided the original work is properly cited and the use is non-commercial. See: http:// creativecommons.org/licenses/by-nc/4.0/

\section{REFERENCES}

1. Munoz A, Nieto F. Cohort studies. New York: Oxford University Press, 2009.

2. Szklo M. Population-based cohort studies. Epidemiol Rev 1998;20:81-90.

3. http://www.nationale-kohorte.de/index_en.html (accessed 20 Nov 2013).

4. Wichmann HE, Kaaks R, Hoffmann W, et al. The German National Cohort. Bundesgesundheitsblatt Gesundheitsforschung Gesundheitsschutz 2012;55:781-7. 
5. Bradburn N. Presidential address: a response to the nonresponse problem. Public Opin Q 1992;56:391-7.

6. Curtin R, Presser S, Singer E. Changes in telephone survey nonresponse over the past quarter century. Public Opin $Q$ 2005;69:87-98.

7. Galea S, Tracy M. Participation rates in epidemiologic studies. Ann Epidemiol 2007;17:643-53.

8. Groves R. Survey errors and survey costs. Hoboken NJ: John Wiley \& Sons, 2004

9. Nohr EA, Frydenberg M, Henriksen TB, et al. Does low participation in cohort studies induce bias? Epidemiology 2006;17:413-18.

10. Boeing $\mathrm{H}$, Korfmann A, Bergmann M. Recruitment procedures of EPIC-Germany. Ann Nutr Met 1999;43:205-15.

11. Hara $M$, Higaki $Y$, Imaizumi $T$, et al. Factors influencing participation rate in a baseline survey of a genetic cohort in Japan. J Epidemiol 2010;20:40-5.

12. Porta $\mathrm{M}$, Gasull $\mathrm{M}$, Puigdomènech $\mathrm{E}$, et al. Sociodemographic factors influencing participation in the Barcelona Health Survey study on serum concentrations of persistent organic pollutants. Chemosphere 2009;76:216-25.
13. Census database of the Census. Munich: Federal Statistical Offices, 2011. https://ergebnisse.zensus2011.de/\#dynTable (accessed 20 Mar 15).

14. Schenk L, Kurth B. Migrantenspezifisches Teilnahmeverhalten an bundesweiten Gesundheitssurveys. Pretestergebnisse und Konsequenzen für die Hauptphase des Kinder- und Jugendgesundheitssurveys (KiGGS). Gesundheitswesen 2004;8/ 9:37-66.

15. Schenk L. Migrant-specific respondent hurdles and access opportunities in the national health interview and examination survey for children and adolescents. Gesundheitswesen 2002;64: 59-68.

16. Winkler V, Leitzmann M, Obi N, et al. Response in individuals with and without foreign background and application to the National Cohort in Germany: which factors have an effect? Int J Public Health 2014;59:555-63.

17. Sinicrope PS, Patten CA, Morgenthaler SB, et al. Healthy women's motivators and barriers to participation in a breast cancer cohort study: a qualitative study. Ann Epidemiol 2009:19: 484-93. 